\title{
Developing an Electron Density Profiler over Europe Based on Space Radio Occultation Measurements
}

\author{
Haris Haralambous and Harris Papadopoulos \\ Electrical Engineering and Computer Science and Engineering Departments \\ Frederick University, 7 Y. Frederickou St., Palouriotisa, Nicosia 1036, Cyprus \\ $\{$ H.Haralambous, H. Papadopoulos\} afrederick.ac.cy
}

\begin{abstract}
This paper presents the development of an Artificial Neural Network electron density profiler based on electron density profiles collected from radio occultation (RO) measurements from LEO (Low Earth Orbit) satellites to improve the spatial and temporal modeling of ionospheric electron density over Europe. The significance in the accurate determination of the electron density profile lies on the fact that the electron density at each altitude in the ionosphere determines the refraction index for radiowaves that are reflected by or penetrate the ionosphere and therefore introduces significant effects on signals (navigation and communication). In particular it represents a key driver for total electron content model development necessary for correcting ionospheric range errors in single frequency GNSS applications.
\end{abstract}

Keywords: Ionosphere, radio occultation, electron density profile.

\section{Introduction}

In recent times the ionospheric monitoring capability has been significantly enhanced on the basis of a multi-instrument approach in both local and regional scale. Traditionally ionospheric monitoring was carried out by ground-based radars (ionosondes) that provide information on the electron density profile (EDP) within a limited geographical area. There have also been examples of ionospheric services in several parts of the globe where the geographical scope of ionosondes has been extended by combining their measurements therefore improving their spatial validity to facilitate provision of maps of ionospheric parameters [1,2,3]. During the last fifteen years ground-based monitoring capability has been significantly augmented by spacebased systems like satellite radio occultation (RO) missions such as CHAMP, and FORMOSAT-3/COSMIC which have increased the spatial scope of these networks.

This paper explores the possibility of utilising this space-based source of ionospheric monitoring with the aim to express the spatial and temporal representation of electron density in the ionosphere over a significant part of Europe. Sections 2 and 3 describe the basic altitude structure of the EDP and its measurement techniques respectively. Section 4 discusses the EDP spatial and temporal characteristics and section 5 outlines the experimental results. Finally, section 6 gives the concluding remarks of the paper. 


\section{Measurement of the Ionospheric Electron Density Profile}

The ionosphere is defined as a region of the earth's upper atmosphere where sufficient ionisation exists to affect radio waves in the frequency range 1 to $3 \mathrm{GHz}$. It ranges in height above the surface of the earth from approximately $50 \mathrm{~km}$ to $1000 \mathrm{~km}$. The influence of this region on radio waves is accredited to the presence of free electrons.

The impact of the ionosphere on communication, navigation, positioning and surveillance systems is determined by variations in its EDP and subsequent electron content along the signal propagation path [4]. As a result satellite systems for communication and navigation, surveillance and control that are based on transionospheric propagation may be affected by complex variations in the ionospheric structure in space and time leading to degradation of the accuracy, reliability and availability of their service.

The EDP of the ionosphere (Figure 1) represents an important topic of interest in ionospheric studies since its integral with altitude determines a very important parameter termed as the total electron content which is a direct measure of the delay imposed on trans-ionospheric radiowaves. The bottomside (below the electron density peak) component of the EDP has been routinely monitored by ionosondes compiling an extended dataset of key profile characteristics on a global scale. A significant subset of these measurements (extending over the last three decades) has been provided by the Global Ionospheric Radio Observatory (GIRO) using the ground-based Digisonde network [5].

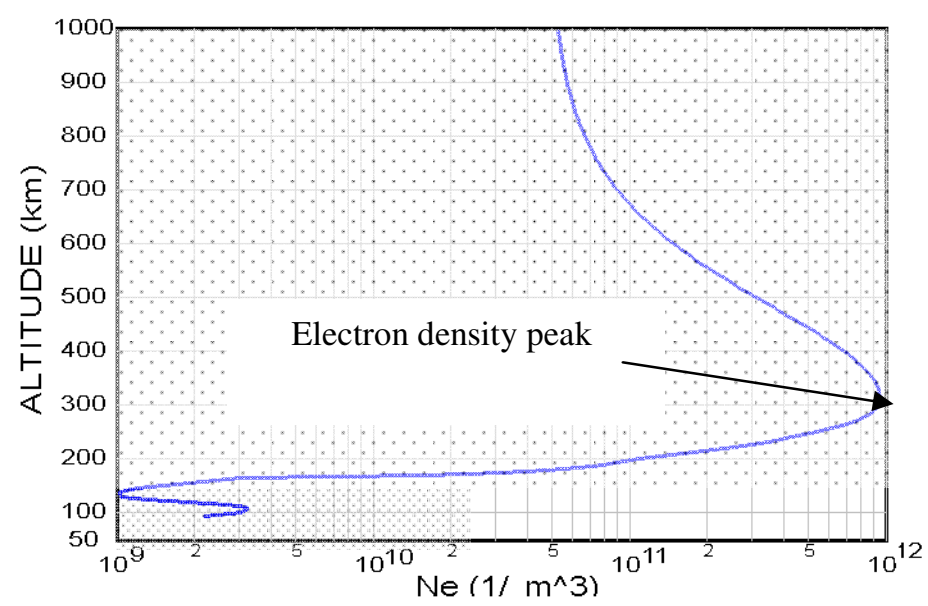

Fig. 1. Typical electron density altitude profile of the ionosphere

The EDP of the topside (above the electron density peak) ionosphere represents an important topic of interest in ionospheric studies since it has been shown that the main contribution to TEC is attributed to an altitude range above the electron density peak. However there is a lack of topside observational data as ground-based ionosondes can probe only up to the electron density peak, and observations from topside sounders 
are sparse since only a few satellite missions, for example Alouette, ISIS-1 and ISIS-2, have been dedicated to topside EDP measurements in the past [6]. The shape of the profile depends upon the strength of the solar ionising radiation which is a function of time of day, season, geographical location and solar activity $[7,8,9]$. This paper studies the development of an Artificial Neural Network (ANN) model which describes the temporal and spatial variability of the EDP over a significant part of Europe. The model is developed based on approximately 80000 LEO satellite EDPs from RO measurements recorded from April 2006 to December 2012.

\section{Measurement of Electron Density by Ground-Based and Satellite Techniques}

Traditionally measurements of electron density were conducted by ionosondes which are special types of radar used for monitoring the electron density at various altitudes in the ionosphere up to the electron density peak. Their operation is based on a transmitter sweeping through the HF frequency range transmitting short pulses. These pulses are reflected at various layers of the ionosphere, and their echoes are received by the receiver giving rise to a corresponding plot of reflection altitude against frequency which is further analysed to infer the ionospheric plasma height-EDP (Figure 2).

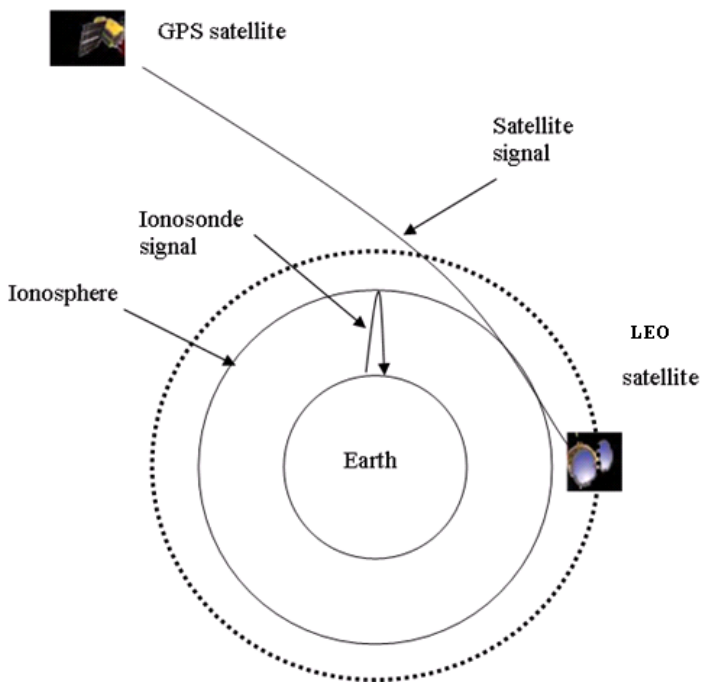

Fig. 2. Schematic illustrating a ground-based (ionosonde) and a space-based technique (satellite $\mathrm{RO})$ for probing the ionosphere

Radio occultation satellite missions of Low Earth Orbit (LEO) satellites are now being widely used for ionospheric studies as they offer an excellent tool for enhancing the spatial aspect of ionospheric monitoring providing information on the vertical 
electron density distribution on a global scale. An important LEO satellite mission used for RO ionospheric measurements is FORMOSAT-3/COSMIC (a constellation of six satellites, called the Formosa Satellite 3-Constellation Observing System for Meteorology, Ionosphere, and Climate) launched on April 15, 2006 [10,11,12]. The instrument on these satellites that is of interest in this paper is the GPS receiver which is used to obtain atmospheric and ionospheric measurements through phase and Doppler shifts of radio signals. The Doppler shift of the GPS L-band ( $\mathrm{L}_{1}=1575.42$ $\mathrm{MHz}, \mathrm{L}_{2}=1227.60 \mathrm{MHz}$ ) signals received by a LEO satellite is used to compute the amount of signal bending that occurs as the GPS satellite sets or rises through the earth's atmosphere as seen from LEO (Figure 2). The bending angles are related to the vertical gradients of atmospheric and ionospheric refractivity which is directly proportional to ionospheric electron density above $80 \mathrm{~km}$ altitude. Through the assumption of spherical symmetry, EDPs can be retrieved from either the bending angles or the total electron content data (computed from the $\mathrm{L}_{1}$ and $\mathrm{L}_{2}$ phase difference) obtained from the GPS RO [13]. We also need to emphasise that the RO technique can be applied successfully in retrieving the ionospheric EDP only under the assumption of spherical symmetry in the ionosphere. This assumption is not always satisfied due to significant electron density gradients that give rise to horizontal electron fluxes. This violates the requirement for EDP inversion producing a very unrealistic profile. In order to overcome this limitation and concentrate on good quality EDPs a selection process was applied in order to exclude those measurements where the distortion of the profiles was excessive [14].

Figure 3 demonstrates the uniform distribution of locations where electron density measurements have been recorded during one week of RO. We can verify the uniform geographical sampling they provide therefore complementing the limited spatial, but high temporal sampling rate (as low as $5 \mathrm{~min}$ ) of the Cyprus ionosonde station (also shown in Figure 3). Measurements from the latter were used to validate the proposed ANN profiler.

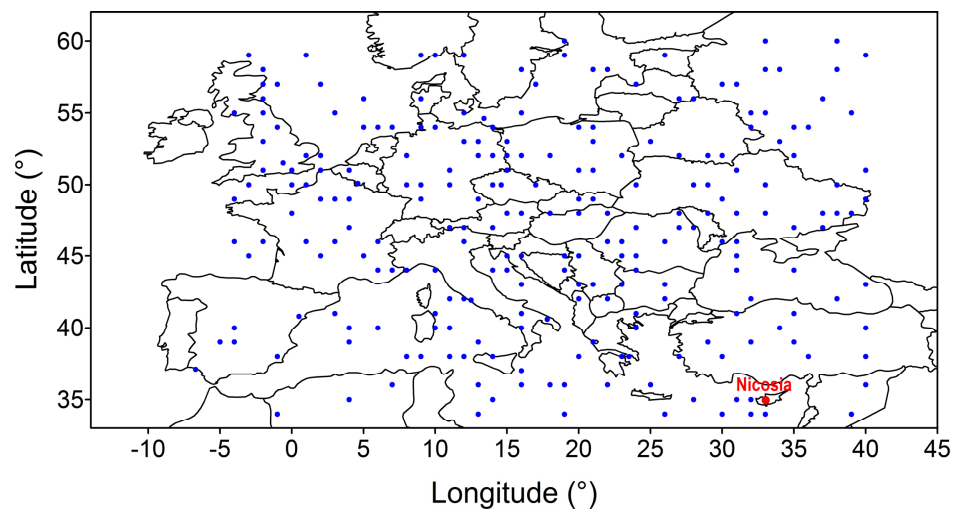

Fig. 3. Map of Europe illustrating the area considered in the model development with positions of one week of RO electron density measurements and location of Cyprus ionosonde station 


\section{Temporal and Spatial Characteristics of Electron Density and Model Parameters}

The temporal variability of the maximum electron density at a single location is well established and has been thoroughly described in previous papers $[15,16]$ primarily based on ionosonde derived electron density datasets. In short, ionospheric dynamics are governed principally by solar activity which in turn influences the electron density of the ionosphere. The EDP exhibits variability on daily, seasonal and long-term time scales in response to the effect of solar radiation. It is also subject to abrupt variations due to enhancements of geomagnetic activity following extreme manifestations of solar activity disturbing the ionosphere from minutes to days on a local or global scale. The most profound solar effect on maximum electron density is reflected on its daily variation as shown in Figures 4 and 5.

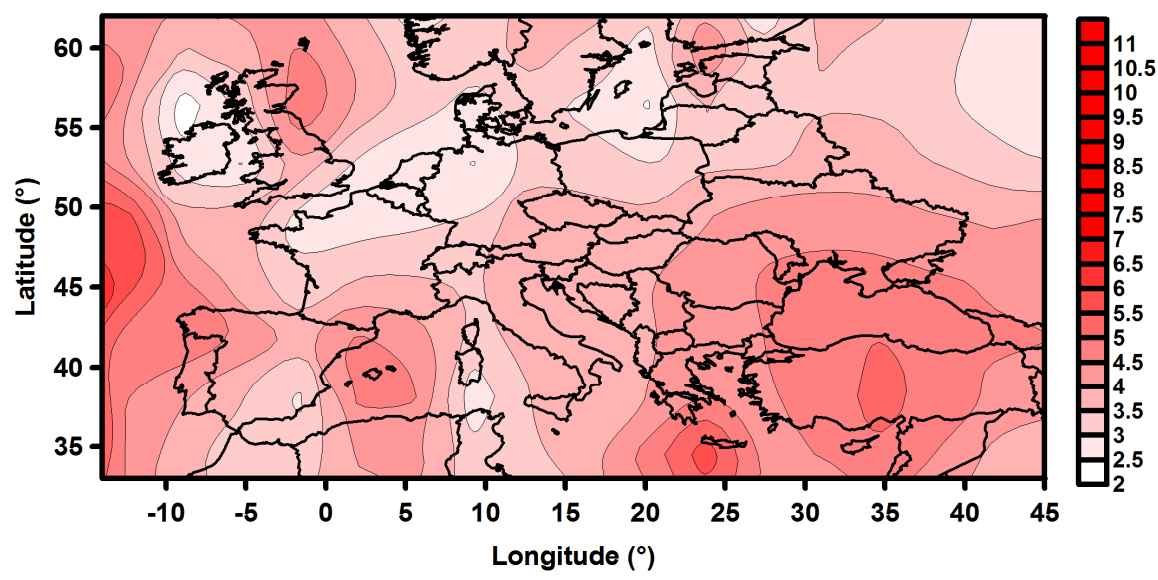

Fig. 4. Global RO maximum electron density map at midnight (universal time-UT)

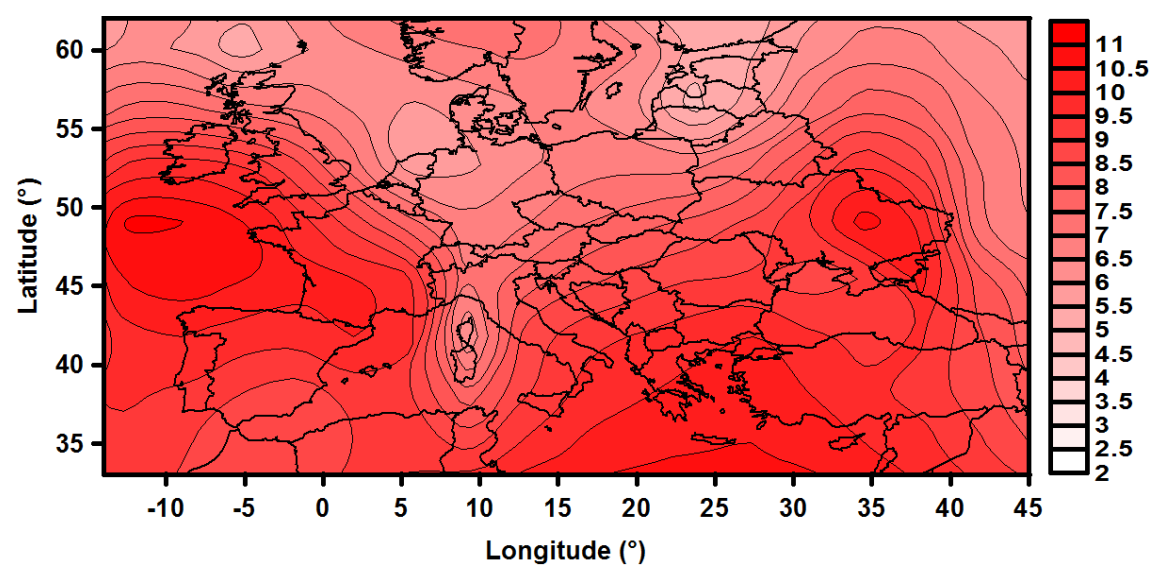

Fig. 5. Global RO maximum electron density map at noon (universal time-UT) 
These figures show a map obtained by the superposition of all maximum electron density values obtained around midnight (Figure 4) and noon (Figure 5) with reference to universal time. As it is clearly depicted, there is a strong dependency of maximum electron density which minimises (over Europe) during the night and maximizes around noon emphasising the strong local time dependence of electron density. This is attributed to the rapid increase in the production of electrons due to the photo-ionization process during the day and a gradual decrease due to the recombination of ions and electrons during the night. This is also evident in Figure 6 where a number of EDPs at different times during a day is shown.

The long-term effect of solar activity on the EDP follows an eleven-year cycle and as it is clearly shown by characteristic examples of EDPs obtained over Cyprus around noon (UT) in Figure 7. Clearly electron density levels are lowest during minimum solar activity (indicated by an index of solar activity termed solar flux $\mathrm{SF}=70$ ) conditions as compared to electron density levels during maximum solar activity conditions $(\mathrm{SF}=130)$.

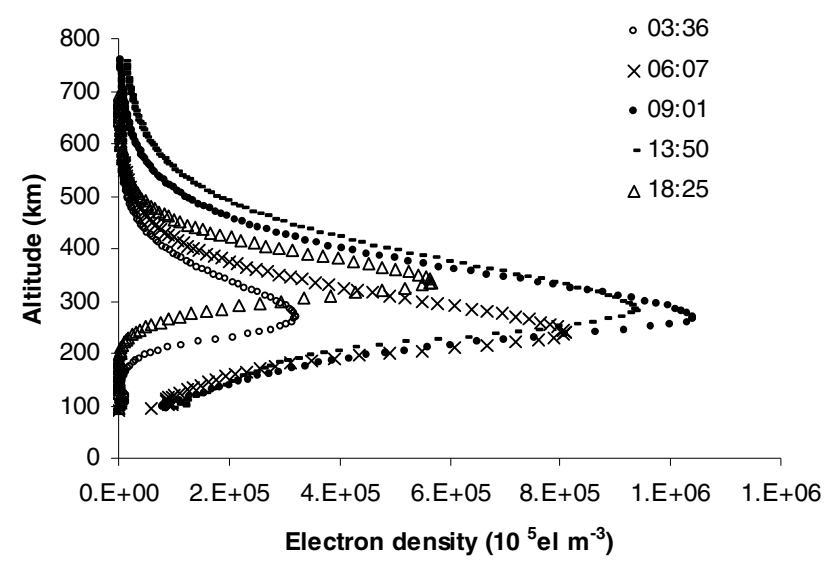

Fig. 6. Examples of EDPs over Cyprus at different hours

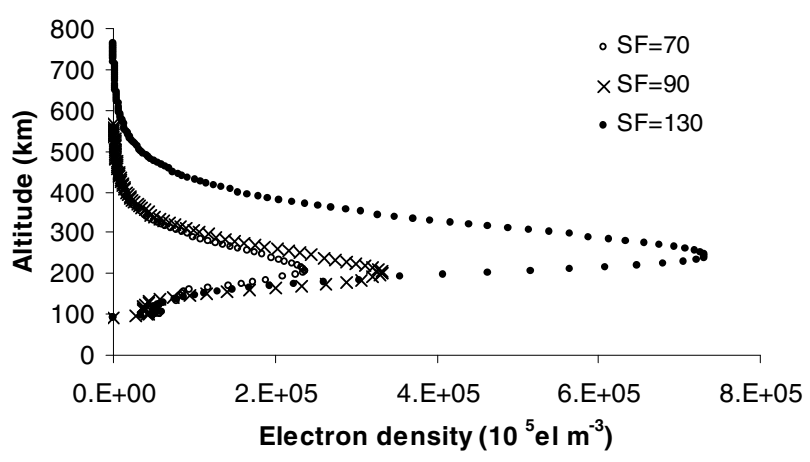

Fig. 7. Examples of EDPs at noon over Cyprus at different solar activity conditions 
In addition to the short-term (diurnal) and long-term (solar cycle) effect on EDP we can also identify a clear spatial effect which is registered in the map shown in Figure 5 as decreasing levels with increasing latitude. This is also depicted in Figure 8(a) where all foF2 (maximum signal frequency that can be reflected by the maximum electron density peak - foF2 is proportional to the square root of the maximum electron density) values obtained from RO measurements are plotted as a function of their latitude (positive latitude is along North). It is evident from this figure that not only the average levels but also the variability in maximum electron density is increased as latitude decreases. This spatial characteristic of diminishing maximum electron density with increasing latitude is also observed in Figure 8(b) where the seasonal variation of the median level of Figure 8(a) (for RO foF2 values obtained over Europe at noon) over the three latitude regimes (low, medium and high) is plotted.

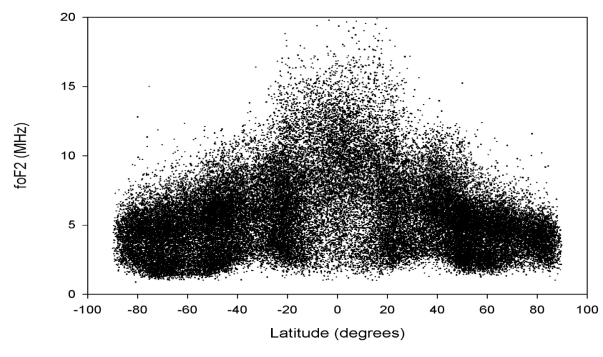

(a)

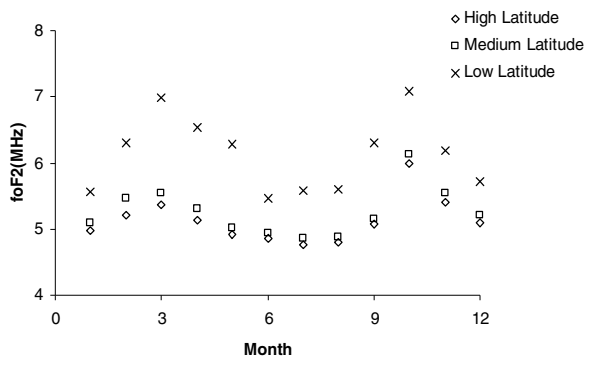

(b)

Fig. 8. (a) foF2 measured by RO versus latitude (b) seasonal variation of foF 2 at noon at low, medium and high latitudes

The plots in Figures 4-8 describe the variabilities that typically characterise the average temporal behaviour of ionospheric electron densities. The model parameters to describe these variabilities have been established in previous papers $[15,16]$ and are annual and daily sinusoidal components as well as a solar activity index (here we use measured daily solar flux). In addition, the year was also used as a temporal parameter as well as latitude and longitude, which express the spatial variability in EDP. Finally, as each EDP corresponds to a number of electron density measurements at different altitudes in the ionosphere, the altitude of each measurement was also used as a parameter; so in effect the resulting model can predict the electron density values at different altitudes and EDP is in fact a set of these predictions.

\section{$5 \quad$ Experiments and Results}

As mentioned in Section 2, approximately 80000 LEO satellite EDPs from RO measurements recorded between April 2006 and December 2012 were used for our experiments. The ANN used had a fully connected two-layer structure, with 9 input and 1 output neurons. Both their hidden and output neurons had hyperbolic tangent sigmoid activation functions. The training algorithm used was the LevenbergMarquardt backpropagation algorithm with early stopping based on a validation set 
created from $20 \%$ of the training examples. In an effort to avoid local minima three ANNs were trained with different random initialisations and the one that performed best on the validation set was selected for being applied to the test examples. The inputs and target outputs of the network were normalized setting their minimum value to -1 and their maximum value to 1 . This made the impact of all inputs in the model equal and transformed the target outputs to the output range of the ANN activation functions. The results reported here were obtained by mapping the outputs of the network for the test examples back to their original scale.

First a 2-fold cross-validation process was followed to examine the performance of the proposed approach on the satellite measurements and choose the best number of hidden units to use. Specifically the dataset was randomly divided in two parts consisting of approximately the same number of EDPs and the predictions of each part were obtained from an ANN trained on the other one. Note that the division of the dataset was done in terms of EDPs (groups of values) and not in terms of individual values. The results of this experiment are reported in Table 1 in the form of the Root Mean Squared Error (RMSE) and Correlation Coefficient (CC) between the predicted and true values over the whole dataset.

After the first experiment the proposed approach was further evaluated by training an ANN with 45 hidden units on the whole dataset and assessing its performance on measurements obtained from Cyprus ionosonde station.

Table 1. The Root Mean Squared Error (RMSE) and Correlation Coefficient (CC) between the predicted and true values over the whole dataset

\begin{tabular}{ccc}
\hline Hidden & RMSE & CC \\
\hline 10 & 67715 & 0.9280 \\
15 & 65385 & 0.9330 \\
20 & 63466 & 0.9370 \\
25 & 62530 & 0.9389 \\
30 & 62189 & 0.9396 \\
35 & 61862 & 0.9403 \\
40 & 62312 & 0.9394 \\
$\mathbf{4 5}$ & $\mathbf{6 1 0 6 5}$ & $\mathbf{0 . 9 4 1 9}$ \\
50 & 61903 & 0.9402
\end{tabular}

The RMSE value as shown in Table 1 lies between 60000 and $6800010^{5} \mathrm{el} \mathrm{m}$. The table clearly demonstrates superior performance for 45 hidden units. However, we must keep in mind that RMSE is just an average measure of the discrepancy between COSMIC and ANN profiler EDP which varies significantly with altitude. Therefore this RMSE value encapsulates different altitude regimes (bottomside, peak and topside) for the proposed ANN profiler into a single value which could be considered as an over-simplification. 


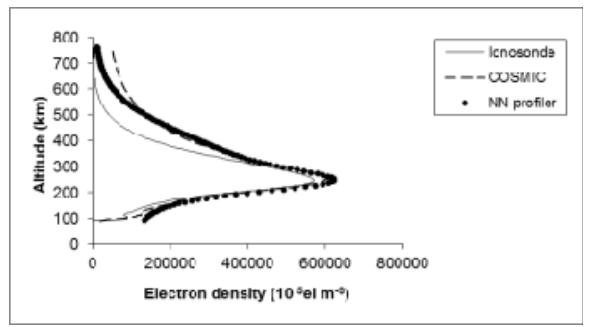

(a)

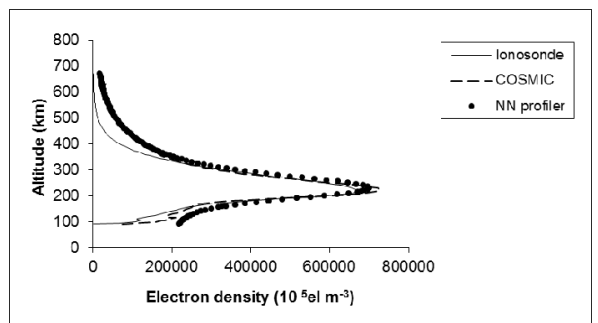

(c)

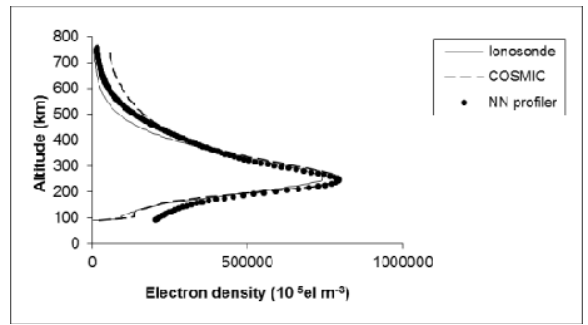

(b)

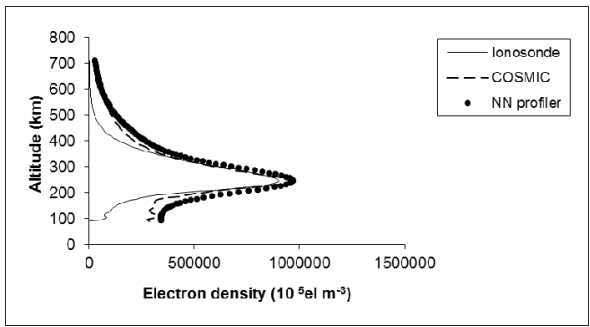

(d)

Fig. 9. Examples of measured (by ionosonde and COSMIC) and predicted (by the ANN profiler) EDPs over Cyprus

The examples of measured ionosonde, COSMIC and ANN profiler EDPs shown in Figure 9 demonstrate the good agreement between measurements and modeled values. The ionosonde and COSMIC profiles in these examples were obtained over Cyprus which was used as a validation point over the region considered in the model development (Figure 3). These particular ionosonde and COSMIC profiles where selected so that their peak electron density and corresponding altitude only differed by less than $5 \%$. In this way we could ensure very good quality profiles in the validation since they were measured by two independent techniques in such a good agreement. We also need to emphasize that in some cases (Figure 9(d)) although the ionosonde and COSMIC EDP profile match very well at the peak they significantly divert at the bottomside. This is due to the presence of very high electron densities in the E-region $(100-130 \mathrm{~km})$ due to long-lived metallic ions forming extremely high-ionisation patches. This phenomenon although termed sporadic-E, is quite frequent over Cyprus sometimes causing difficulties in the inversion of occultation measurements into a meaningful EDP around these altitudes. We also need to note that in the case of an ionosonde EDP the topside is actually modeled by a special extrapolation function based on the measured bottomside EDP. This explains the difference with COSMIC EDPs at the topside above $300-400 \mathrm{~km}$.

\section{Conclusions}

In this paper we have presented the development of an ANN electron density profiler based on satellite electron density profiles to improve the spatial and temporal modeling of ionospheric electron density over Europe. The profiler exhibited promising prospects in profiling electron density over Europe over time and space. 


\section{References}

1. Belehaki, A., Cander, L., Zolesi, B., Bremer, J., Juren, C., Stanislawska, I., Dialetis, D., Hatzopoulos, M.: DIAS project: The establishment of a European digital upper atmosphere server. Journal of Atmospheric and Solar-Terrestrial Physics 67(12), 1092-1099 (2005)

2. Wilkinson, P., Patterson, G., Cole, D.G., et al.: Australian space weather services-past and present. Adv. Space Res. 26(1), 233-236 (2000)

3. Cander $\mathrm{Lj}, \mathrm{R}$.: Towards forecasting and mapping ionosphere space weather under cost actions. Adv. Space Res. 31(4), 4957-4964 (2003)

4. Barclay, L.W.: Ionospheric Effects and Communication Systems performance. In: Keynote paper at the 10th Ionospheric Effects Symposium, Washington DC (2002)

5. Reinisch, B.W., Galkin, I.A., Khmyrov, G.M., Kozlov, A.V., Bibl, K., Lisysyan, I.A., Cheney, G.P., Huang, X., Kitrosser, D.F., Paznukhov, V.V., Luo, Y., Jones, W., Stelmash, S., Hamel, R., Grochmal, J.: The new digisonde for research and monitoring applications. Radio Sci. 44, RS0A24 (2009)

6. Reinisch, B.W., Huang, X.: Deducing topside profiles and total electron content from bottomside ionograms. Adv. Space Res. 27, 23-30 (2001)

7. Goodman, J.: HF Communications, Science and Technology. Nostrand Reinhold (1992)

8. Maslin, N.: The HF Communications, A Systems Approach, San Francisco (1987)

9. McNamara, L.F.: Grid The Ionosphere: Communications, Surveillance, and Direction Finding. Krieger Publishing Company, Malabar (1991)

10. Schreiner, W., Rocken, C., Sokolovsky, S., Syndergaard, S., Hunt, D.: Estimates of the precision of GPS radio occultations from the COSMIC/FORMOSAT-3 mission. Geophys. Res. Lett. 34, L04808 (2007), doi:10.1029/2006GL027557

11. Rocken, C., Kuo, Y.-H., Schreiner, W., Hunt, D., Sokolovsky, S., McCormick, C.: COSMIC system description, Terr. Atmos. Ocean Sci. 11, 21-52 (2000)

12. Wickert, J., Reigber, C., Beyerle, G., König, R., Marquardt, C., Schmidt, T., Grunwaldt, L., Galas, R., Meehan, T.K., Melbourne, W.G., Hocke, K.: Atmosphere sounding by GPS radio occultation: First results from CHAMP, Geophys. Res. Lett. 28, 3263-3266 (2001)

13. Hajj, G.A., Romans, L.J.: Ionospheric electron density profiles obtained with the Global Positioning system: Results from the GPS/MET experiment. Radio Sci. 33, 175-190 (1998)

14. Yang, K.F., Chu, Y.H., Su, C.L., Ko, H.T., Wang, C.Y.: An examination of FORMOSAT3/COSMIC F peak and topside electron density measurements: data quality criteria and comparisons with the IRI model. Terr. Atmos. Ocean Sci. 20, 193-206 (2009)

15. Haralambous, H., Papadopoulos, H.: A Neural Network Model for the Critical Frequency of the F2 Ionospheric Layer over Cyprus. In: Palmer-Brown, D., Draganova, C., Pimenidis, E., Mouratidis, H. (eds.) EANN 2009. CCIS, vol. 43, pp. 371-377. Springer, Heidelberg (2009)

16. Haralambous, H., Ioannou, A., Papadopoulos, H.: A neural network tool for the interpolation of foF2 data in the presence of sporadic E layer. In: Iliadis, L., Jayne, C. (eds.) EANN/AIAI 2011, Part I. IFIP AICT, vol. 363, pp. 306-314. Springer, Heidelberg (2011) 